\title{
Cytogenetic mapping of the Lethal hybrid rescue gene of Drosophila simulans
}

\author{
Masa-Toshi Yamamoto*, Masaki Kamo, Setsuko Yamamoto, and Takao K. Watanabe \\ Department of Applied Biology, Kyoto Institute of Technology, Matsugasaki, Sakyo, Kyoto 606, Japan
}

(Received 29 September 1997, accepted 25 November 1997)

\begin{abstract}
Hybrids not carrying Drosophila simulans X chromosome derived from the cross between $D$. melanogaster females and $D$. simulans males are lethal at the late third instar larval period or early pupal stage. This lethality can be rescued by the mutation Lethal hybrid rescue ( $L h r$ ) of $D$. simulans. The Lhr gene is considered to play an important role in reproductive isolation, but the genetic analyses have not been carried out extensively because of the lack of visible mutations and chromosome rearrangements in $D$. simulans. Using a cuticle mutation, jabara, of which locus is close to $L h r$, and $D$. melanogaster deficiencies, we performed cytological mapping in hybrids and estimated the $L h r$ locus to $54 \mathrm{E}-\mathrm{F}$.
\end{abstract}

\section{INTRODUCTION}

Viability of hybrids between Drosophila melanogaster females and $D$. simulans males differs dramatically depending on the sex. The hybrid females are viable, although sterile, but the males do not survive to the adult flies. They develop up to the third instar larval stage, and then live as larvae for about three weeks and eventually die as third instar larvae or pharate pupae (Yamamoto, 1992; Sawamura et al., 1993b). This lethality is due to the absence of $D$. simulans $\mathrm{X}$ chromosome in the hybrids, for females carrying a compound $\mathrm{X}$ chromosome (attached-X) of $D$. melanogaster also die at the same stage of the development. No effect of $D$. simulans Y chromosome on the hybrid viability has been demonstrated (Yamamoto, 1992). Reciprocal crosses, $D$. simulans females and $D$. melanogaster males, produce viable male hybrids but lethal females.

The most conventional genetic approach to understand the biological mechanism of the hybrid lethality is to find the genes which rescue the lethal hybrids, and let them develop to the adulthood. So far four genes have been reported as hybrid rescue genes, which are Lethal hybrid rescue (Lhr) and maternal hybrid rescue ( $m h r)$ of D. simulans, and Hybrid male rescue (Hmr) and zygotic hybrid rescue (zhr) of D. melanogaster (Watanabe, 1979; Hutter and Ashburner 1987; Hutter et al., 1990; Sawamura et al., 1993a, 1993b, 1993c). The two genes, $L h r$ and $m h r$, of D. simulans have not been analyzed as extensively as $\mathrm{Hmr}$ and $z h r$ of D. melanogaster.

In order to understand the genetic mechanisms by molecular terms, it is the basic requirement to map the gene location on the chromosomes. In $D$. simulans, however,

\footnotetext{
* Corresponding author.
}

available visible marker genes and chromosome rearrangements are so few that genetic analyses as extensive as in D. melanogaster are impossible to be done. The Lethal hybrid rescue gene ( $L h r)$ of $D$. simulans was the first rescue gene among the four (Watanabe, 1979), but no detailed genetic analyses had been carried out. The gene was roughly mapped genetically to 95 on the second chromosome, but the location may not be accurate because the mapping was done by using visible markers, black (b) and plum ( $\mathrm{pm}$ ), both are widely separated from $L h r$, and the number of recombinants examined was relatively small. To map the $L h r$ locus more precisely, we combined genetic mapping of a novel cuticle mutation in $D$. simulans and cytological mapping using hybrids carrying deficiencies of D. melanogaster. The location of $L h r$ is estimated to the salivary bands $54 \mathrm{E}-\mathrm{F}$.

\section{MATERIALS AND METHODS}

Fly stocks. The following stocks were used and flies were bred on the standard cornmeal-yeast-glucose-agar medium at $22-23^{\circ} \mathrm{C}$.

D. simulans stocks: The following mutant stocks were used; $L h r, L h r$ pm, net b py sd pm, and jabara (jba). As the wild type strain RM1 (an isofemale line of Rakujuen) was used. For phenotypic descriptions, see Sturtevant (1929).

D. melanogaster stocks: $y w$ and the following second chromosome deficiencies were used; $D f(2 R) \mathrm{Jp} 1$ (51C3; 52F5-9), $D f(2 R) P c 4$ (55A; 55F), $D f(2 R) P c l 7 B$ (54E8-F1; 55B9-C1), $D f(2 R) P c l 11 B$ (54F6-55A1; 55C1-3), $D f(2 R) P 34$ (55E2; 56B2,C1), Df (2R)AA21 (56F9-17; 57D11-12), Df (2R)PI13 (57B13-14; 57D8-9), Df (2R)PuD17 (57B4; 58B), and $D f(2 R) P K 1$ (57C5;57F5-6). For descriptions of genes 
and chromosome rearrangements, see Lindsley and Zimm (1992), Castrillon et al. (1993), and Török et al. (1997). $D f(2 R) P 34$ was the gift from Peter Bryant, University of California, Irvine, and the other deficiency stocks were kindly provided by Umeå Stock Center and Bloomington Stock Center.

Polytene chromosome analysis. Salivary glands of third instar larvae were dissected out in $0.7 \% \mathrm{NaCl}$ solution and then transferred to $2 \%$ lacto-acetic orcein. After ten min, the salivaries were squashed and examined under the phase-contrast microscope.

\section{RESULTS AND DISCUSSION}

Phenotype of jabara mutation. A spontaneous cuticle mutation jabara ( $j b a$ ) was found in an isofemale line collected in Takefu, Japan by Saito in 1992. The fly has a bellows-like appearance in tergites as shown in Figure 1. They look bulgy in abdominal segments. The both anterior and posterior peripheries of the dark pigmented bands of tergites appear to be tucked inside the epidermis. The tuck lines, shown in Figure 1B and C, are clearly observed under the stereomicroscope. The phenotype is conspicuous in the second to fifth tergites, but very weak in the sixth tergite. The tucked-in cuticle can be extended by pressing the abdomen, suggesting the cuticle component is left intact. Actually the extended cuticle structure of jabara appears normal when observed under the microscope. The phenotype is very easy to identify and to discriminate from the wild type. Age apparently enhances the phenotype, but the newly emerged flies can be classified without overlap with the wild type. The mutation should be classified as RK1.

Genetic mapping of $\boldsymbol{L h} \boldsymbol{r}$ and $\boldsymbol{j} \boldsymbol{j} \boldsymbol{b}$. Preliminary genetic mapping of $j b a(2-90)$ and the previous preliminary data of $L h r(2-95)$ (Watanabe, 1972) suggest that the loci are both in the region between $s d$ and $p m$, which are known to be at 80 and 103 in the map, respectively (Sturtevant, 1929). Thus we conducted genetic mapping of $L h r$ and $j b a$ using $s d$ and $p m$.

Females heterozygous for net b py sd pm and $L h r$ were backcrossed to net b py sd pm males, and recombinant males whose phenotype was either $s d$ or $p m$ were selected. Hereafter the chromosome region including net $b$ and $p y$ will not be considered since they are far distant from the region in question. In order to identify males carrying either $L h r$ or $L h r^{+}, \mathrm{F}_{1}$ males were crossed individually to two to three $y w$ females of $D$. melanogaster, and counted the number of hybrid males and females. As summarized in Table 1, $1219 \mathrm{pm}$ and $458 \mathrm{sd}$ males were examined, and respective 394 and 11 males produced hybrids. Occurrence of hybrid males among the progeny indicates that the parental male carried $L h r$. As results, four classes of gametes
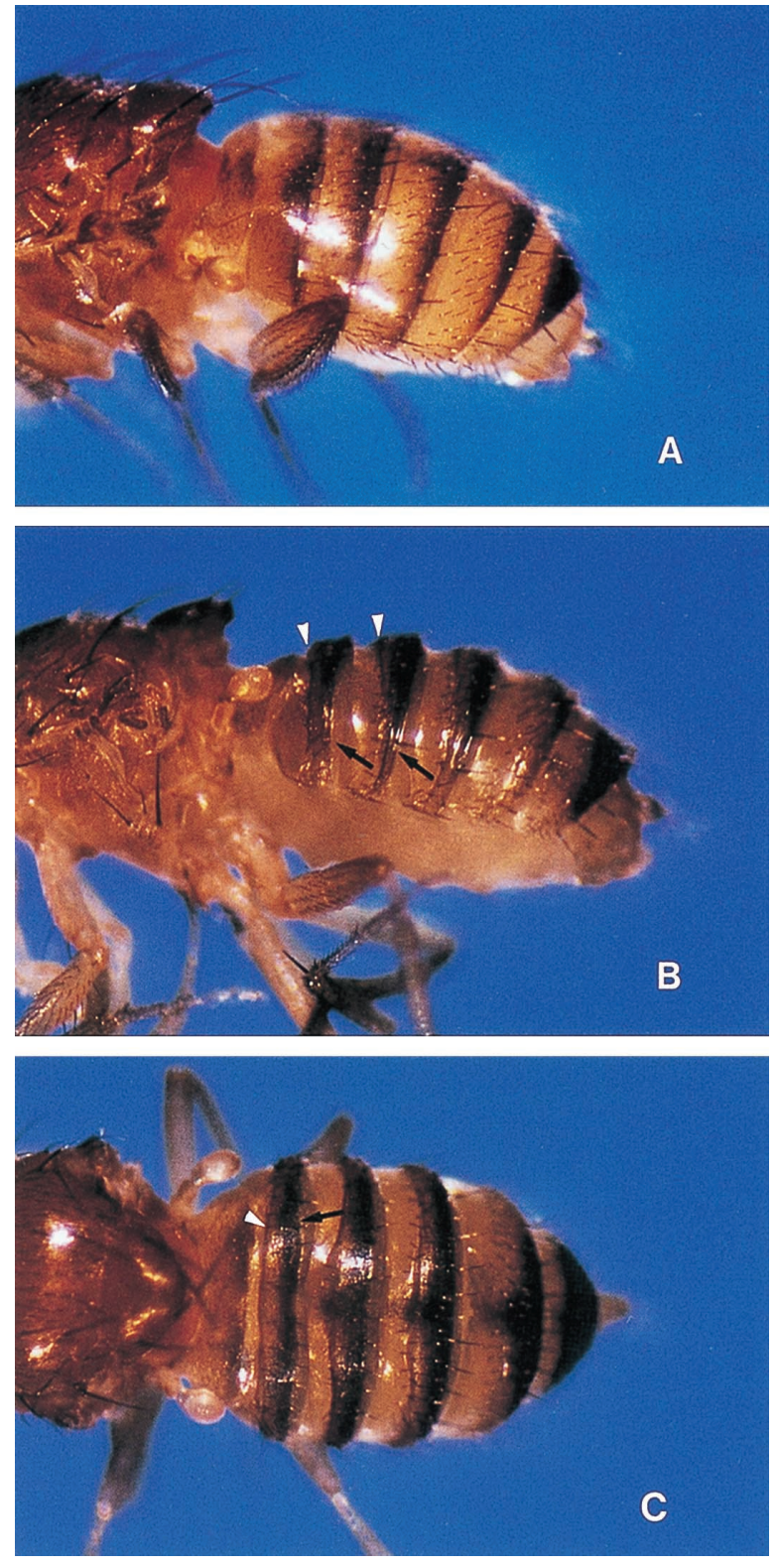

Fig. 1. Abdominal tergite morphology of the wild type female of Drosophila simulans (A), and jabara mutant (B and C). White arrow head and black arrow indicate the anterior and posterior cuticle tuck lines, respectively, in a tergite $(\mathrm{B}$, side view and $\mathrm{C}$, dorsal view).

were identified: Recombinants between $s d$ and $L h r$ were 4 sd $\mathrm{Lhr} \mathrm{pm}^{+}$and $144 \mathrm{sd}^{+} \mathrm{Lhr}^{+} \mathrm{pm}$, and those between $\mathrm{Lhr}$ and $p m$ were $7 s d L h r^{+} p^{+}$and $250 s d^{+} L h r p m$. Thus the $L h r$ locus is estimated to be at the position $36.5 \%$ apart from $s d$ between $s d$ and $p m$. Extrapolating from the genetic map of $s d$ (80) and $p m$ (103), $L h r$ should be mapped at 88.4 .

As can be seen in Table 1, the crossability of the $\mathrm{pm}$ males to $D$. melanogaster females was $32.3 \%$, while the $s d$ males only $2.4 \%$. It should be noted that the $p m$ males were in- 
Table 1. Results of genetic mapping of $L h r$

\begin{tabular}{|c|c|c|c|c|c|c|}
\hline $\begin{array}{c}\text { Phenotype } \\
\text { of } \\
\text { recombinant }\end{array}$ & $\begin{array}{l}\text { Number of } \\
\text { crosses } \\
\text { set up }\end{array}$ & $\begin{array}{l}\text { Number of } \\
\text { crosses } \\
\text { succeeded }\end{array}$ & $\begin{array}{c}\text { Number of } \\
\text { lines producing } \\
\text { hybrid males }\end{array}$ & $\begin{array}{c}\text { Number of } \\
\text { lines not } \\
\text { producing } \\
\text { hybrid males }\end{array}$ & $\begin{array}{c}\text { Genotype } \\
\text { of } \\
\text { gamete }\end{array}$ & $\begin{array}{c}\text { Region } \\
\text { of } \\
\text { exchange* }\end{array}$ \\
\hline \multirow{2}{*}{$\begin{array}{l}\text { plum } \\
\text { eye } \\
\text { color }\end{array}$} & \multirow[b]{2}{*}{1219} & \multirow[b]{2}{*}{394} & 250 & & Lhr pm & \\
\hline & & & & 144 & $+p m$ & \\
\hline \multirow{2}{*}{$\begin{array}{l}\text { spread } \\
\text { wings }\end{array}$} & \multirow{2}{*}{458} & \multirow{2}{*}{11} & 4 & & $s d L h r$ & \\
\hline & & & & 7 & $s d+$ & \\
\hline
\end{tabular}

Only recombinat males from the cross, $s d \mathrm{pm} / L \mathrm{hr}$ females and $s d \mathrm{pm}$ males, were used to cross D. melanogaster females. For details see text.

* Two regions at which recombination occurred are indicated as , region between $s d$ and Lhr; , region between $L h r$ and $p m$.

dividually crossed to D. melanogaster females and $32.3 \%$ of them produced hybrids. However, when we used $s d$ males individually for the crosses, all 120 males examined failed to produce hybrids. To solve the problem of this low crossability, we set up the following second lot of experiments. We first expanded the number of males of the same genotype as their father who is under examination whether carrying $L h r$ or $L h r^{+}$. A $s d$ male was crossed individually to net $b$ py sd pm females and $\mathrm{F}_{1}$ males showing $s d \mathrm{pm}^{+}$phenotype were selected. Ten males from an expanded lines as described above were used for crosses to ten females of D. melanogaster. A total of 338 lines of $s d$ males were examined by this procedure in the second lot. Yet, only 11 of them gave hybrids. The difference in crossability between the two groups (plum eye color and spread wing males) could have resulted from their viability difference as a whole. However, a significantly low mating success rate in the males of black body color and spread wings suggests that the held out wings might cause insufficient wing vibration in the mating behavior. It should be pointed out that the genetic mapping was not affected by the difference in crossability because the recombination frequencies obtained from the two groups were identical.

We mapped jba after constructing a $s d j b a$ pm stock. Because there is no balancer chromosome in $D$. simulans, first we made a cross between net $b$ py sd pm and jba, and then backcrossed to net $b$ py $s d$ pm males. From the $\mathrm{F}_{1}, b$ py sd females were picked up and were crossed to net $b$ py $s d$ pm males, and $b$ py sd jba pm females and males were selected to maintain as a homozygous stock. Females from the stock were crossed to the wild type males and $F_{1}$ females were backcrossed to $s d$ jba pm males, and recombinant flies were scored as shown in Table 2. The exchange frequencies in the regions $s d-j b a$ and $j b a-p m$ were measured as $44 \%$ and $56 \%$, respectively. The location of $j b a$ is thus at 90.1, which is 1.7 map units distal to the Lhr locus. Possible variation in genetic map units has been minimized by the use of common stock net by $s d \mathrm{pm}$ in both experi-
Table 2. Number of flies produced from the cross, $s d j b a \mathrm{pm} /$ +++ females and $s d j b a$ pm males for genetic mapping of the jabara locus

\begin{tabular}{ccccc}
\hline \hline \multicolumn{2}{c}{ Region } & \multicolumn{2}{c}{ Region } & Total \\
\cline { 1 - 3 }$s d++$ & $+j b a p m$ & $s d j b a+$ & $++p m$ & \\
\cline { 1 - 3 } 280 & 211 & 360 & 268 & 1119 \\
\hline
\end{tabular}

Regions at which recombination occurred are indicated as , region between $s d$ and $j b a ; \quad$, region between $j b a$ and $p m$.

ments.

Cytogenetic mapping of $\mathbf{j b a}$ and $\mathbf{p m}$. In order to map the cytological location of the $L h r$ gene, we used deletion chromosomes of $D$. melanogaster and made hybrids with $j b a$ or $\mathrm{pm}$. The deletions used and the results are summarized in Figure 2.

The eye color phenotype of pm appeared in the hybrids bearing the deficiency $D f(2 R) A A 21, D f(2 R) P I 13$, $D f(2 R) P u D 17$, or $D f(2 R) P K 1$, indicating that the $p m$ gene is within the region between 57C5 and 57D8-9. The corresponding gene of $D$. melanogaster is likely to be Punch $(\mathrm{Pu})$ whose locus is 57C (McLean et al., 1990; Sugaya and Fuyama, 1995).

The cuticle mutation $j b a$ was also examined similarly using four deficiencies available between the divisions 54 to 56 , that were $D f(2 R) P c 4, D f(2 R) P c l 7 B, D f(2 R) P c l 11 B$, and $D f(2 R) P 34$. As shown in Figure 2, three deficiencies uncovered the $j b a$ mutation. The cytological location is thus determined to be 55A1;55B9-C1.

Location of $\mathbf{L h r}$ on the salivary map. The cytological localization of $j b a$ and $p m$ provides clues to estimate the location of the $L h r$ locus. Genetic distances should be proportional to the cytological distance especially in the middle portion of a chromosome arm (Lindsley and Sandler, 1977). The genetic map distance between $L h r$ and $j b a$ is 12.9 , and 


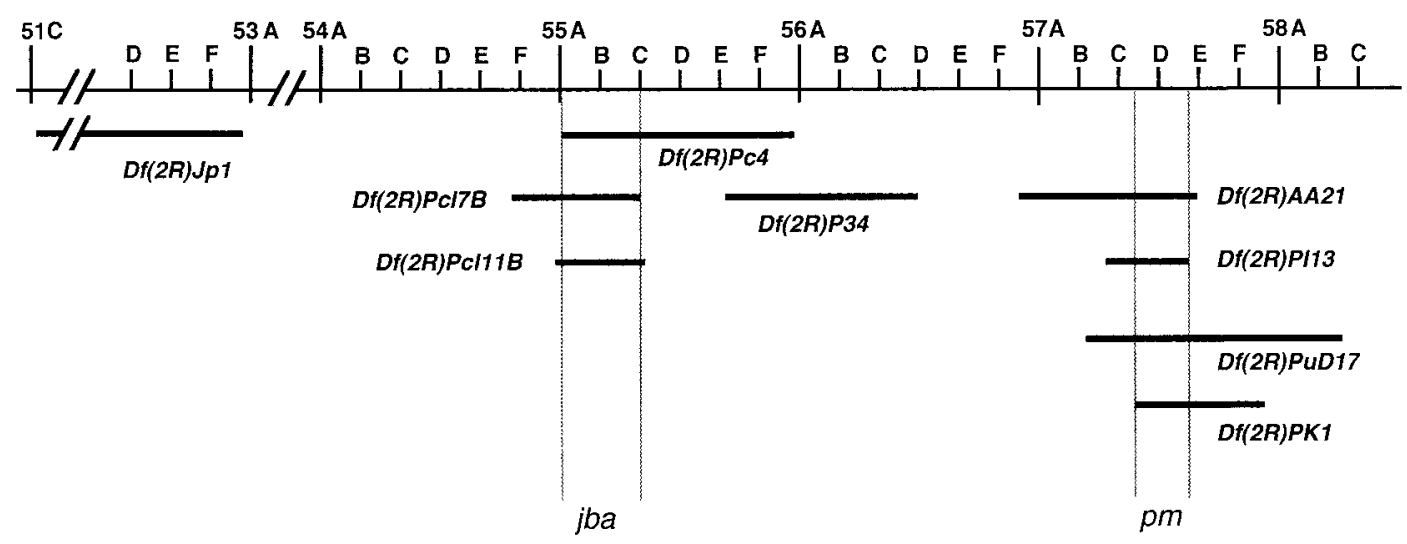

Fig. 2. Second chromosome deficiencies of $D$. melanogaster used for the cytological mapping of $j b a$ and $p m$. The salivary map is illustrated on the top line with division numbers and subdivisions. Each bar shows the size and location of the deficiency.

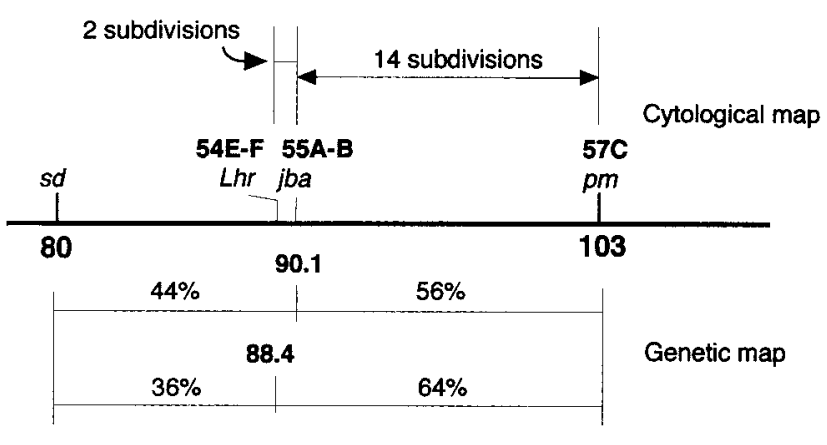

Fig. 3. Summary of the genetic and cytological mapping of $j b a$ and $L h r$. Genetic map of $j b a$ and $L h r$ was determined by recombination frequency between the two genes, $s d$ and $p m$. Cytological locations of $L h r$ was estimated from the genetic data and cytological results of $j b a$ and $p m$. For details, see the text.

the number of salivary map subdivisions between the two genes is 14. These map distances give an estimate of the cytological location of the $L h r$ locus. The genetic map distance of $L h r$ from $p m$ is 14.6, which should be equal to about 16 subdivisions. Therefore the $L h r$ locus on the salivary map is estimated to be at $54 \mathrm{E}-\mathrm{F}$.

Similarly the $s d$ locus is expected to be located at 53C-D. We do not know what gene of $D$. melanogaster corresponds to $s d$, but hybrids carrying $s d$ and the balancer chromosome $C y O$ of the $D f(2 R) J p 1 / C y O$ stock provided from the Umeå Stock Center showed the same wing phenotype as $s d$.

We have no clue to identify what gene of $D$. melanogaster is equivalent to $L h r$, but from the cytological mapping described here we have narrowed down the chromosomal site, we must look at in order to search for the gene of $D$. melanogaster. Mutations in the candidate gene would be expected to show analogous phenotypes to the lethal hybrids, such as prolonged larval period before death and abnormal chromosome condensation (Yamamoto, 1994; Orr et al., 1997).
This work was partly supported by Grant-in-Aid for Scientific Research (A) from the Ministry of Education, Science, Sports, and Culture of Japan.

\section{REFERENCES}

Castrillon, D. H., Gönczy, P., Alexander, S., Rawson, R., Eberhart, C. G., Viswanathan, S., DiNardo, S., and Wasserman, A. (1993) Toward a molecular genetic analysis of spermatogenesis in Drosophila melanogaster: Characterization of male-sterile mutants generated by single $P$ element mutagenesis. Genetics 135, 489-505.

Hutter, P., and Ashburner, M. (1987) Genetic rescue of inviable hybrids between Drosophila melanogaster and its sibling species. Nature 327, 331-333.

Hutter, P., Roote, J., and Ashburner, M. (1990) Molecular analysis of a candidate gene for the reproductive isolation between sibling species of Drosophila. Genetics 124, 909-920.

Lindsley, D. L., and Sandler, L. (1977) The genetic analysis of meiosis in female Drosophila melanogaster. Phil. Trans. R. Soc. Lond. B. 277, 295-312.

Lindsley, D. L., and Zimm, G. G. (1992) The Genome of Drosophila melanogaster. Academic Press, San Diego.

McLean, J. R., Boswell, R., and O'Donnell, J. (1990) Cloning and molecular characterization of a metabolic gene with development functions in Drosophila. I. Analysis of the head function of Punch. Genetics 126, 1007-1019.

Orr, H. A., Laurence, D. M., Coyne, J. A., Goodwin, R., and Hawley, R. S. (1997) The developmental genetics of hybrid inviability: A mitotic defect in Drosophila hybrids. Genetics 145, 10311040.

Sawamura, K., and Yamamoto, M-T. (1993) Cytogenetical localization of Zygotic hybrid rescue $(\mathrm{Zhr})$, a Drosophila melanogaster gene that rescues interspecific hybrids from embryonic lethality. Mol. Gen. Genet. 239, 441-449.

Sawamura, K., Taira, T., and Watanabe, T. K. (1993a) Hybrid lethal systems in the Drosophila melanogaster species complex. I. The maternal hybrid rescue $(\mathrm{mhr})$ gene of Drosophila simulans. Genetics 133, 299-305.

Sawamura, K., Watanabe, T. K., and Yamamoto, M-T. (1993b) Hybrid lethal systems in the Drosophila melanogaster species complex. Genetica 88, 175-185.

Sawamura, K., Yamamoto, M-T., and Watanabe, T. K. (1993c) Hybrid lethal systems in the Drosophila melanogaster species complex. II. The Zygotic hybrid rescue $(\mathrm{Zhr})$ gene of $D$. 
melanogaster. Genetics 133, 307-313.

Sturtevant, A. H. (1929) The genetics of Drosophila simulans. Publication No. 399 of Carnegie Institution of Washington, pp. 1-62. Washington, D. C.

Sugaya, S., and Fuyama, Y. (1995) pm of D. simulans is homologous to $P u$ of D. melanogaster. Drosophila Inf. Serv. 76, 81.

Török, T., Harvie, P. D., Buratovich, M., and Bryant, P. J. (1997) The product of proliferation disrupter is concentrated at centromeres and required for mitotic chromosome condensation and cell proliferation in Drosophila. Genes Dev. 11, 213225 .
Watanabe, T. K. (1979) A gene that rescues the lethal hybrids between Drosophila melanogaster and D. simulans. Jpn. J. Genet. 54, 325-331.

Yamamoto, M-T. (1992) Inviability of hybrids between $D$. melanogaster and $D$. simulans results from the absence of simulans $\mathrm{X}$ not the presence of simulans $\mathrm{Y}$ chromosome. Genetica 87, 151-158.

Yamamoto, M-T. (1994) Hybrid inviability and hybrid rescue genes. Tanpakushitsu Kakusan Koso 39, 2660-2670. (in Japanese). 